\title{
Quasi-One-Dimensional Intermittent Flux Behavior in Superconducting Films
}

\author{
A. J. Qviller, ${ }^{1}$ V. V. Yurchenko, ${ }^{1}$ Y. M. Galperin,,${ }^{1,2,3}$ J. I. Vestgården, ${ }^{1}$ P. B. Mozhaev,,${ }^{4,5}$ \\ J. B. Hansen, ${ }^{4}$ and T. H. Johansen ${ }^{1,3,6}$ \\ ${ }^{1}$ Department of Physics, University of Oslo, P.O. Box 1048 Blindern, 0316 Oslo, Norway \\ ${ }^{2}$ Ioffe Physical Technical Institute of the Russian Academy of Sciences, 194021 St. Petersburg, Russia \\ ${ }^{3}$ Centre for Advanced Study at the Academy of Science and Letters, 0271 Oslo, Norway \\ ${ }^{4}$ Department of Physics, Technical University of Denmark, Kongens Lyngby, DK-2800, Denmark \\ ${ }^{5}$ Institute of Physics and Technology of the Russian Academy of Sciences, Moscow, 117218, Russia \\ ${ }^{6}$ Institute for Superconducting and Electronic Materials, University of Wollongong, \\ Northfields Avenue, Wollongong, New South Wales 2522, Australia
}

(Received 16 June 2011; published 27 January 2012)

Intermittent filamentary dynamics of the vortex matter in superconductors is found in films of $\mathrm{YBa}_{2} \mathrm{Cu}_{3} \mathrm{O}_{7-\delta}$ deposited on tilted substrates. Deposition of this material on such substrates creates parallel channels of easy flux penetration when a magnetic field is applied perpendicular to the film. As the applied field is gradually increased, magneto-optical imaging reveals that flux penetrates via numerous quasi-one-dimensional jumps. The distribution of flux avalanche sizes follows a power law, and data collapse is obtained by finite-size scaling, with the depth of the flux front used as crossover length. The intermittent behavior shows no threshold value in the applied field, in contrast to conventional flux jumping. The results strongly suggest that the quasi-one-dimensional flux jumps are of a different nature than the thermomagnetic dendritic (branching) avalanches that are commonly found in superconducting films.

DOI: 10.1103/PhysRevX.2.011007

Subject Areas: Materials Science, Statistical Physics, Superconductivity

Since the prediction of a regular vortex lattice in type-II superconductors [1], a wide variety of vortex systems has been thoroughly investigated $[2,3]$. The pronounced dependencies of the range and strength of the vortex-vortex interaction on temperature and magnetic field make the vortex matter a unique, tunable model system for probing the statistical properties of interacting systems. The behavior of the vortex matter is to a large extent also determined by its interaction with quenched disorder in the material, i.e., with microscopic defects that pin the vortices and therefore serve as pinning centers in the superconductor. The interplay between the pinning forces and the driving Lorentz force leads to formation of a metastable critical state [4], where the current density has a critical magnitude, $j_{\mathrm{c}}$, which corresponds to the maximal nondissipative current density.

In films of $\mathrm{YBa}_{2} \mathrm{Cu}_{3} \mathrm{O}_{7-\delta}$ (YBCO) grown epitaxially, i.e., with proper matching of crystal lattice parameters, on a substrate slightly tilted from a major crystal plane (vicinal films), self-organized arrays of planar defects (antiphase boundaries) are formed with a typical period of 2-5 nm [5]. The terrace structure of the surface is characterized by steps that are a few Ångströms in height, and the steps provide well-oriented seeding sites for growth

Published by the American Physical Society under the terms of the Creative Commons Attribution 3.0 License. Further distribution of this work must maintain attribution to the author(s) and the published article's title, journal citation, and DOI. along the main axis ( $c$ axis) of the overlying YBCO film. Films grown on substrates with a tilt angle of $\theta$ close to $10^{\circ}$ have better grain alignment and improved current-carrying ability $[5,6]$. There has been considerable interest in such films due to their potential application in Josephsonjunction design [7-9], in particular for highly sensitive detectors of electromagnetic radiation.

The growth of YBCO films on tilted substrates is also known to produce planar defects, which become channels for easy vortex motion $[5,6,10]$. These channels are separated by $5-10 \mu \mathrm{m}$ and run parallel to the terrace structure, causing considerable anisotropy in both the flux penetration and the effective critical current. At low temperatures, where the anisotropy is most pronounced, the vortices are essentially confined inside the channels, and the flux penetration has a strong quasi-one-dimensional (quasi-1D) character. In this paper, we show, using magneto-optical imaging (MOI), that the quasi-1D flux dynamics is highly intermittent at low temperatures in samples with moderate tilt angles.

Intermittent flux motion is very harmful for electronic devices, so the source of intermittency should be identified and the behavior characterized. Until now, intermittent flux behavior in superconducting films has been observed in $\mathrm{Nb}, \mathrm{Pb}, \mathrm{Nb}_{3} \mathrm{Sn}, \mathrm{NbN}, \mathrm{MgB}_{2}$, and $\mathrm{YNi}_{2} \mathrm{~B}_{2} \mathrm{C}$ [11-16], where, during slow ramping of the applied field, the flux avalanches take the form of large dendritic (branching) structures. These avalanches are believed to be caused by a thermomagnetic instability [17-20]. We find that, in YBCO on tilted substrates, the flux jumps are largely 


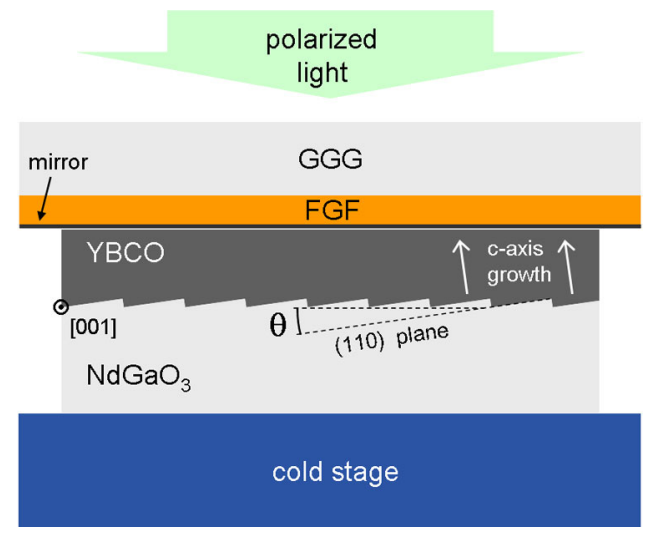

FIG. 1. Mounting in the cryogenic MOI setup of the YBCO film on a $\mathrm{NdGaO}$ substrate with a terrace-structured surface. Also shown is the orientation of the crystallographic axes specified by Miller indices, as well as the tilt angle $\theta$. On top of the superconductor is the Faraday rotating ferrite-garnet film (FGF), grown on a gadolinium-gallium-garnet (GGG) substrate, which allows realtime visualization of the flux density across the film area.
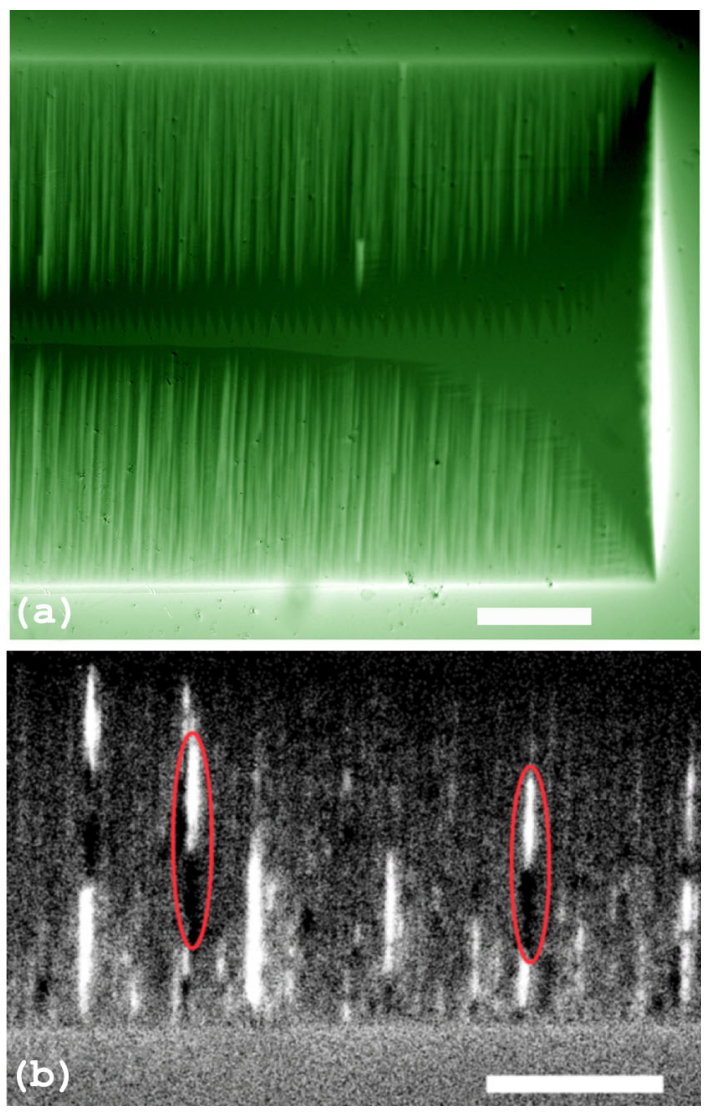

FIG. 2. (a) Direct and (b) differential magneto-optical images of flux penetration in a YBCO film on a $\theta=14^{\circ}$-tilted substrate. The applied magnetic field is $B_{a}=16.2 \mathrm{mT}$, and the sample temperature is $T=4 \mathrm{~K}$. The image brightness represents the distribution of flux density. In (b), the linear streaks show the avalanches taking place during $\Delta B_{a}=42.5 \mu \mathrm{T}$. The white scale bars at the bottom of each panel are $200 \mu \mathrm{m}$ long. different from the thermomagnetic avalanches both in morphology and in size distribution, suggesting that the origin of the intermittency is of a different nature.

Superconducting films are prepared by laser ablation (vaporization by a laser beam and subsequent crystallization) of $\mathrm{YBa}_{2} \mathrm{Cu}_{3} \mathrm{O}_{7-\delta}$ ceramic targets on $\mathrm{NdGaO}_{3}$ substrates with tilt angles $\theta$ of up to $33^{\circ}$. (See Ref. [9] for sample preparation details.) The films we investigate in the present work have thickness of $d=200 \mathrm{~nm}$ and a critical temperature of $T_{c}=88 \mathrm{~K}$. They are shaped by optical lithography into long strips of width $2 w=0.9 \mathrm{~mm}$. Lowtemperature MOI is performed using an in-plane magnetized ferrite-garnet film as the Faraday rotating sensor $[21,22]$. The measurement configuration is illustrated in Fig. 1.

Figure 2(a) shows a magneto-optical image of the flux penetration in a $\theta=14^{\circ}$ film where the surface steps are aligned vertically in the picture. The sample is initially cooled to $T=4 \mathrm{~K}$ at zero field, and then a perpendicular applied magnetic field is slowly increased at a rate of $0.2 \mathrm{mT} / \mathrm{s}$. The image is recorded at $B_{a}=16.2 \mathrm{mT}$. The flux penetrates the sample from the two long edges, seen in the figure as bright horizontal lines, in a distinctly filamentary manner, where the filaments are aligned with the underlying terrace steps. From the short edge, however, the flux penetration is essentially blocked by the strong pinning associated with vortex motion transverse to the terrace structure. The full flux dynamics during such a field ramp is seen in Video 1.

From the real-time observation, it is evident that the penetration is strongly intermittent, where the flux enters from both long edges via discrete quasi-1D avalanches. This penetration is also documented in panel (b) of Fig. 2,

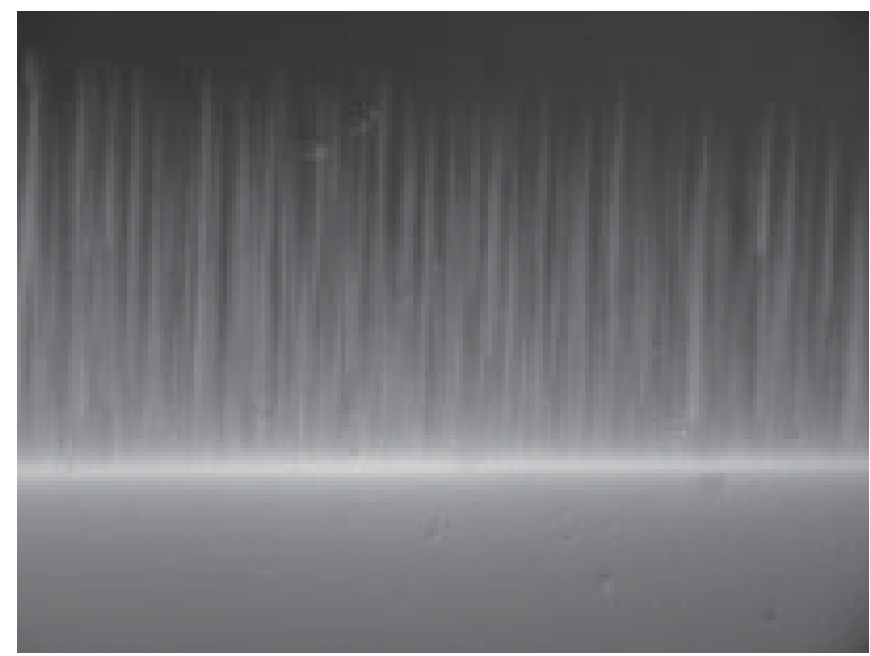

VIDEO 1. The intermittent flux penetration in the YBCO film shown in Figure 2. Needlelike flux avalanches are seen as abrupt events as the magnetic field $B_{a}$ is increased from zero to $17 \mathrm{mT}$ in steps of $\Delta B_{a}=42.5 \mu \mathrm{T}$. The sample temperature is $T=8 \mathrm{~K}$ 


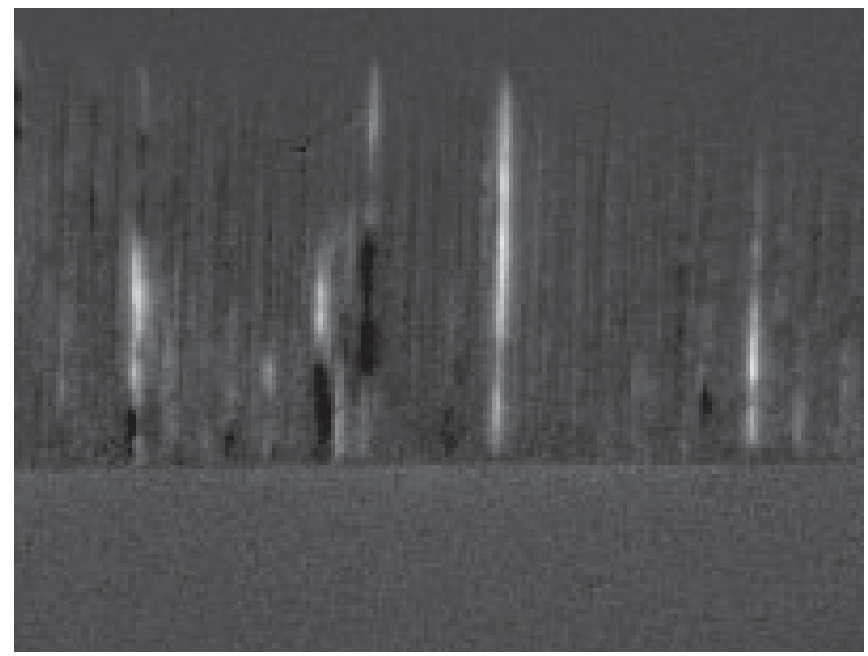

VIDEO 2. The differential video obtained by subtracting subsequent frames in Video 1.

which shows the result of subtracting two subsequently recorded images. The bright streaks show the abrupt arrival of the flux entering either (i) from the edge where new vortices nucleate, or (ii) from an internal vortex rearrangement [23]. The red ovals in the figure mark examples of internal avalanches, whereas the dark streaks are the regions from which the flux escaped. All this dynamics takes place within a field interval of only $\Delta B_{a}=42.5 \mu \mathrm{T}$. A differential movie of the flux dynamics is included as Video 2. Analyzing the sequence of avalanches from such movies, we find no correlation between flux jumps in adjacent channels. Thus, the overall flux-penetration process can be considered as a sequence of independent avalanche events.

More insight into the phenomenon is obtained by determining whether the avalanches are reproducible or not. The well-known dendritic flux avalanches caused by thermomagnetic instability have the characteristics of being irreproducible $[15,24,25]$. When a field sweep is repeated under the same experimental conditions, the flux patterns show essentially no overlap, suggesting that stochastic fluctuations play a main role. The present quasi-1D avalanches also display irreproducible behavior, although on a smaller scale. Figure 3 shows a superposition of three monochrome color-coded (red, green, and blue) differential images taken at the same applied field and temperature during three different consecutive experimental runs. In an image made in this way, repeatable features appear as a gradation of gray for repeatable features, whereas events that occur in only one run appear as red, green, or blue. The superposition is black in areas where no flux change occurs in any of the images. Evidently, there is no overlap in the three sets of flux avalanches [26]. The same irreproducibility has been found at all fields investigated.

All the quasi-1D avalanches are limited in length by the overall flux-penetration front as it advances toward the

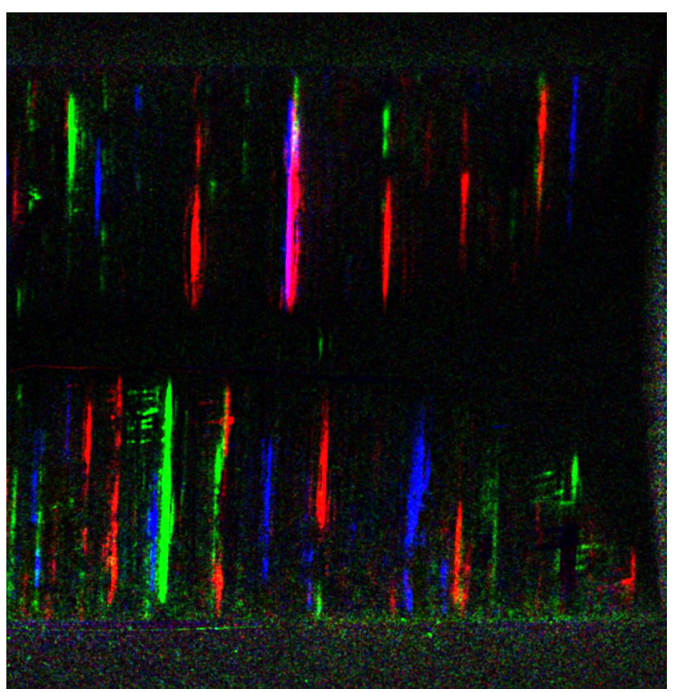

FIG. 3. Superposition of three magneto-optical differential images of flux penetration in a YBCO film on a $\theta=14^{\circ}$-tilted substrate. The different monochrome image series are color coded in red, green, and blue, respectively. The presence of colored superpositions shows that the flux jumps are not reproducible. The sample temperature is $T=4 \mathrm{~K}$, the applied magnetic field is $B_{a}=16.7 \mathrm{mT}$, and the field step is $\Delta B_{a}=$ $42.5 \mu \mathrm{T}$. The scale is the same as for Fig. 2(a).

middle of the strip in response to the increasing applied magnetic field. The front is built up as an envelope of numerous flux needles formed by the avalanches penetrating the most deeply into the film. This result is strikingly different from the dendritic type of avalanches, where the size of the jumps can span the sample even with a small applied field, and the flux front in general attains a very complex shape. In our case, the flux front is far more well behaved.

To quantify the new type of intermittent flux dynamics, we record magneto-optical images while the applied field

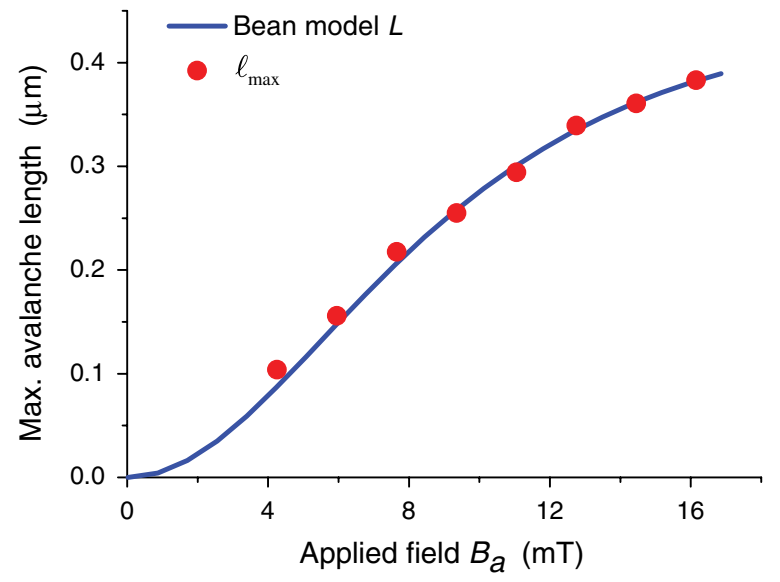

FIG. 4. Dependence of the maximum avalanche length, $\ell_{\max }$, on the applied field (red dots). The solid blue curve shows the Bean-model flux penetration depth, $L$, given by Eq. (1). 


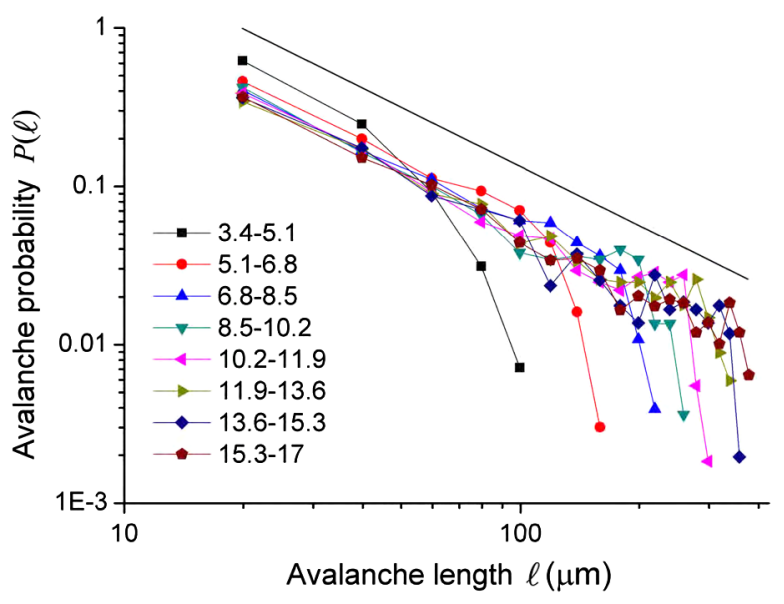

FIG. 5. Statistics of avalanche length, $\ell$, observed while the applied field is ramped from zero to $B_{a}=17 \mathrm{mT}$ in steps of $\Delta B_{a}=42.5 \mu \mathrm{T}$. The curves correspond to the field intervals listed in the key (mT). The straight line with a slope of -1.3 is added as a guide to the eye.

is slowly ramped from zero to $B_{a}=17 \mathrm{mT}$. The sample becomes nearly fully penetrated by flux. Images are recorded every $42.5 \mu \mathrm{T}$. From all these images, a sequence of difference images is prepared, and, for every avalanche that occurs, its length $\ell$ is measured by using a patternrecognition program. Figure 4 shows how $\ell_{\max }$, the size of the longest avalanche within each field interval, varies with the applied field. The maximum length follows a smooth curve, which corresponds very well to the advancement of the overall flux front, i.e., at all fields, there are avalanches starting at the edges and ending at the flux front. Actually, the $\ell_{\max }\left(B_{a}\right)$ behaves in full accord with the fluxpenetration depth for a long thin superconducting strip in the critical state. For a strip in the Bean critical state, the flux front reaches a depth of $L$ given by [27]

$$
L / w=1-\cosh ^{-1}\left(B_{a} / B_{d}\right)
$$

where $B_{d}=\mu_{0} j_{\mathrm{c}} d / \pi$. In Fig. $4, L\left(B_{a}\right)$ is plotted to fit the experimental data using a critical current density of $j_{\mathrm{c}}=$ $0.78 \times 10^{11} \mathrm{Am}^{-2}$ as the only fitting parameter. Based on the good agreement, we conclude that the overall flux front evolves with the applied field according to the conventional critical-state behavior, whereas the detailed dynamics is governed by quasi-1D flux avalanches of length up to the depth of the flux front.

Figure 5 shows the size distribution of all the detected avalanches of more than 10000 , as a normalized probability for the occurrence of an avalanche of length $\ell$. The data are grouped into equal field intervals of $1.7 \mathrm{mT}$. From the $\log -\log$ plot, it is evident that the vast majority of the avalanches are distributed as a power law $P(\ell) \sim \ell^{-\tau}$. The avalanche distribution has a natural cutoff length at the overall flux-penetration depth $L\left(B_{a}\right)$. A fit to the linear part of the data gives $\tau=1.3$ for the exponent. Applying finite-size scaling according to the relation

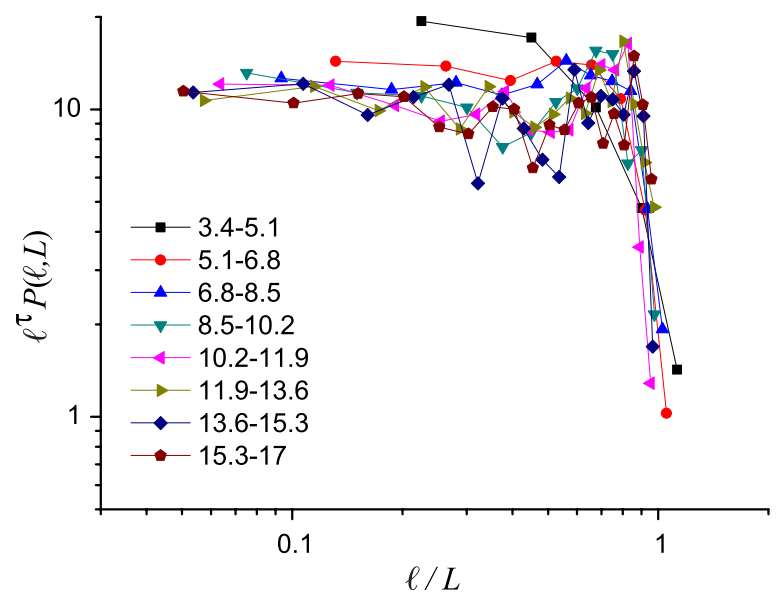

FIG. 6. Collapse of the data in Fig. 5, showing finite-size scaling. The graphs correspond to the field intervals listed in the key $(\mathrm{mT})$.

$$
\mathcal{P}(\ell, L) \propto \ell^{-\tau} f\left(\frac{\ell}{L^{D}}\right)
$$

where $f$ is a scaling function, the plot in Fig. 6 shows that a good data collapse is obtained with $D=1$.

To summarize the experimental results, we have found that, at small fields, the avalanches are also small, and, as the applied field becomes larger, the maximum avalanche size gradually increases. The envelope curve of the fluxpenetration front is slightly ramified (split into branches) by the avalanche activity, but it still behaves essentially in full accord with the critical-state model. Analyzing avalanches that occur within a small field interval as the applied field increases, we find that the distribution of

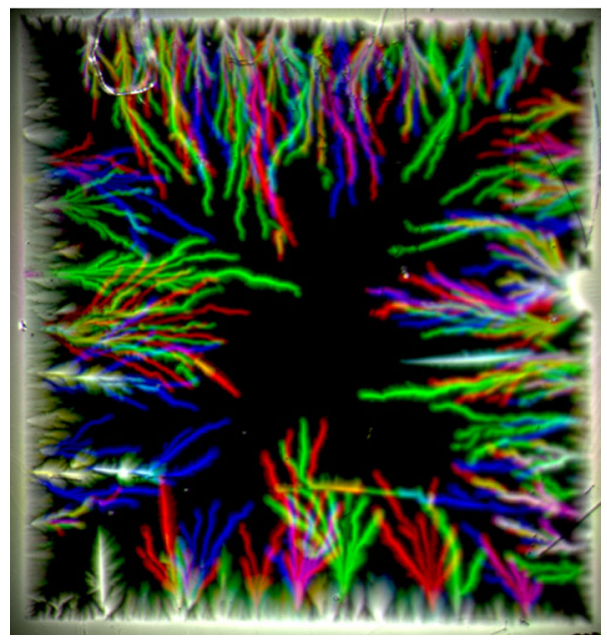

FIG. 7. Superposition of three magneto-optical images (red, green, and blue) taken at the same applied field, $B_{a}=22 \mathrm{mT}$ at $T=9.2 \mathrm{~K}$ in three different experimental runs. The presence of colors shows that the dendritic avalanches are irreproducible. The sample is a $5 \times 5 \mathrm{~mm}^{2}, 300$-nm-thick film of $\mathrm{MgB}_{2}$ on $\mathrm{Al}_{2} \mathrm{O}_{3}$ (1102) substrate. 


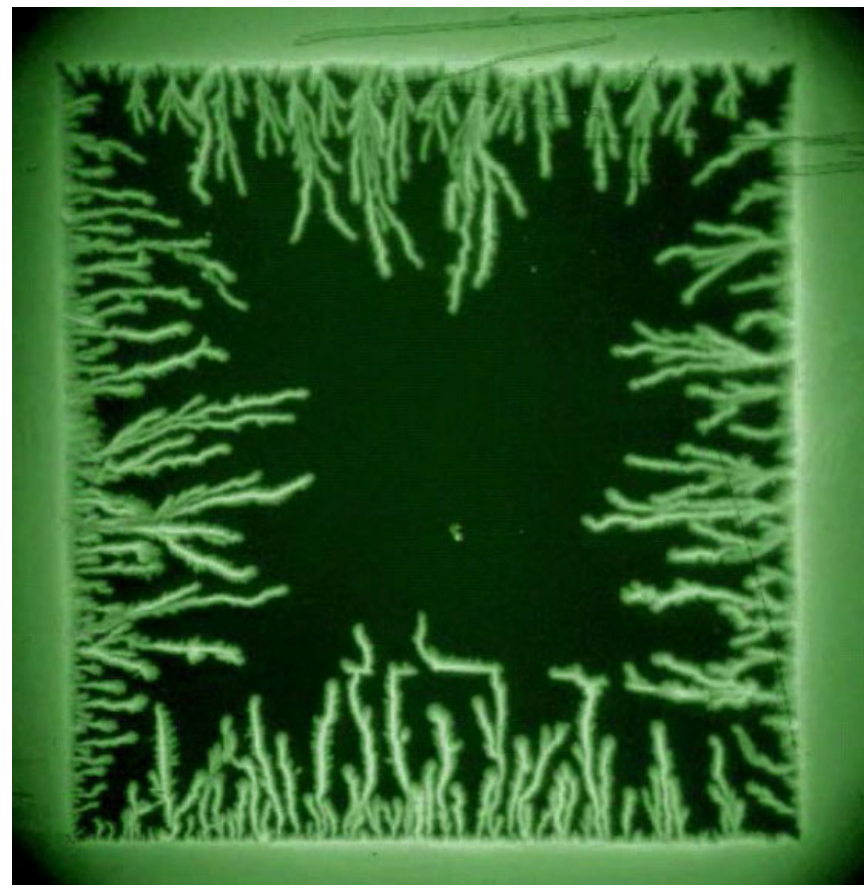

VIDEO 3. For comparison, a typical set of flux avalanches in a $\mathrm{MgB}_{2}$ film. Notice the different morphology between these avalanches spanning the sample in complex dendritic patterns, and those seen in Video 1.

avalanche sizes is a monotonously decreasing curve. All these characteristics are in stark contrast to the observations reported for flux avalanches caused by thermomagnetic runaways. Figure 7 illustrates the typical dendritic morphology of the thermomagnetic-runaway type of avalanches, here seen in a film of $\mathrm{MgB}_{2}$. A movie of the dendritic flux penetration into a film of $\mathrm{MgB}_{2}$ is seen in Video 3. The avalanches protrude in highly nonregular shapes, deep into the Meissner-state part of the film, and histograms of the avalanche size show a strongly peaked distribution [28]. Moreover, in contrast to the dendritic avalanches, our observations of the present YBCO films do not detect any threshold field for onset of the avalanche activity. Thus, there are several significant differences between the two types of avalanches, which suggest that the quasi-1D flux jumps in YBCO on tilted substrates are not of a thermomagnetic origin.

A more likely scenario is that the intermittent filamentary behavior is a result of vortex dynamics under conditions of channeling, which is clearly seen in the current MOI experiments. The film has a large number of linear defects which form parallel narrow channels with low pinning surrounded by areas of strong pinning, thus providing quasi-1D confinement. As flux enters these channels, some vortices will also penetrate into the strongpinning areas, thus forming a fence on both sides. These vortices, which sit at strong-pinning sites, certainly will have substantial positional disorder and hence add a considerable random component to the confinement. Thus, the channels will contain a sequence of random bottlenecks for the traffic of vortices. As the applied magnetic field increases, more and more vortices penetrate into the channels, and situations analogous to a traffic jam, or clogging, will occur. Whenever one of these blocked regions eventually breaks open, an avalanche of vortex motion takes place along the channel. Since these barriers can be arbitrarily small, there is no threshold applied field for the onset of this intermittent behavior. This picture is consistent with our experimental observations. Moreover, this mechanism allows avalanches to start not only from the sample edge, but also from inside the channels, which is also in accordance with our observations. Interestingly, this scenario resembles the flux motion found in artificially created mesoscopic flow channels. (See Ref. [29] and references therein.)

It is also worth discussing our results within the framework of self-organized criticality (SOC). Although the first dynamical system shown to display SOC, the BakTang-Wiesenfeld sand pile [30], does not exhibit SOC in one dimension, several similar algorithms with different toppling rules and intrinsic randomness do [31-33]. These models were invented to study numerically a mechanical quasi-1D dynamical system, the Oslo rice pile [34], which for certain types of rice grains displays SOC behavior. The numerical simulations give power-law avalanche-size distributions with exponents in the range of 1.3-1.6, depending on the details of the model. Our measured avalanche exponent falls within this range.

In the SOC literature, various definitions are used for the size of an avalanche: the number of sites being toppled, the amount of dissipated energy, the spatial extent of an avalanche, etc. In the present work, we have also performed an alternative statistical analysis where the avalanche size is taken to be the amount of the flux being abruptly displaced in each event, rather than the length of the filamentary jumps. This alternative analysis also leads to good scaling and a power-law exponent that agrees within 0.1 of that reported in this paper. Aegerter et al. [35] have also reported power-law statistics of flux avalanches in YBCO thin films deposited on plain substrates. Using MOI, they find the exponent $\tau$ close to ours. However, their observed avalanches are far from being one dimensional, having $D=1.89$. Closer-to-one-dimensional motion has been observed in small bundles of vortices in superconductor films of tungsten by use of scanning microscopy [36]. However, the data were accumulated over periods of many minutes, showing very different time characteristics compared to our observations. Note that, in contrast to the previous fluximaging experiments[35,36], the present MOI observations cover the avalanche activity of the entire flux pile, enabling us to perform finite-size scaling with the physical size of the pile $L$, instead of analyzing a small viewing window.

Worth noting in Fig. 6 is the slight overshoot in the curves near the cutoff length, $L$. Also, simulations 
[31-33] show this feature, i.e., a small increase in the probability of having avalanches of maximum size, which is interpreted as a finite-size effect [31]. To our knowledge, this effect has not been observed experimentally before in vortex systems.

Since inertia can destroy SOC in mechanical systems [37], superconducting vortices, commonly considered as massless, indeed represent a very favorable candidate for SOC, and a lot of numerical simulation work has been reported on the topic [38-41]. In particular, the simulations for two-dimensional vortex motion in a slab [39] show SOC behavior with scaling and power-law distributions, including an overshoot similar to that in our data.

We also perform similar experiments on YBCO films using two other ramp rates: $0.12 \mathrm{mT} / \mathrm{s}$ and $0.02 \mathrm{mT} / \mathrm{s}$. No significant qualitative or quantitative changes in the behavior are observed. Moreover, intermittent flux dynamics has also been observed in vicinal samples with tilt angles of $\theta=8^{\circ}, 10^{\circ}$, and $12^{\circ}$. However, in films with $\theta=3^{\circ}, 20^{\circ}$, $26^{\circ}$, and $33^{\circ}$, we find, not abrupt flux motion, but rather a smooth penetration into the channels. A reference sample with zero-degree tilt angle has shown neither flux jumps nor channeling. More work is required to clarify the relationship between the sample morphology and flux dynamics in superconductor films grown on vicinal-cut substrates.

In conclusion, we find that a gradual injection of the magnetic flux into a system of well-defined channels may trigger quasi-1D vortex avalanches, the sizes of which are distributed by a power law with the avalanche exponent $\tau=1$.3. Introducing the depth of the flux front as a dynamic length scale makes the distributions invariant.

We acknowledge fruitful discussions with V.M. Vinokur, C. Reichhardt, K. T. Tallakstad, K. J. Måløy, J. Bergli, and E. Altshuler. The substrates for the films were produced by I. M. Kotelyanskii. The kind assistance of J. E. Mozhaeva during the sample preparation is also greatly appreciated. This work was financially supported by the Norwegian Research Council.

[1] A. A. Abrikosov, On the Magnetic Properties of Superconductors of the Second Group, Zh. Eksp. Teor. Fiz. 32, 1442 (1957); [On the Magnetic Properties of Superconductors of the Second Group, Sov. Phys. JETP 5, 1174 (1957)].

[2] G. Blatter, M. V. Feigel'man, V.B. Geshkenbein, A. I. Larkin, and V. M. Vinokur, Vortices in High-Temperature Superconductors, Rev. Mod. Phys. 66, 1125 (1994).

[3] E. H. Brandt, The Flux-Line Lattice in Superconductors, Rep. Prog. Phys. 58, 1465 (1995).

[4] C. P. Bean, Magnetization of High-Field Superconductors, Rev. Mod. Phys. 36, 31 (1964).

[5] Ch. Jooss, R. Warthmann, and H. Kronmuller, Pinning Mechanism of Vortices at Antiphase Boundaries in $\mathrm{YBa}_{2} \mathrm{Cu}_{3} \mathrm{O}_{7-\delta}$, Phys. Rev. B 61, 12433 (2000).
[6] A. Polyanskii, R. L. S. Emergo, J.Z. Wu, T. Aytug, D. K. Christen, G. K. Perkins, and D. Larbalestier, MagnetoOptical Imaging and Electromagnetic Study of $\mathrm{YBa}_{2} \mathrm{Cu}_{3} \mathrm{O}_{7}$ Vicinal Films of Variable Thickness, Phys. Rev. B 72, 174509 (2005).

[7] J.H. Kim and D. Youm, Growth of Textured c-Axis Normal $\mathrm{YBa}_{2} \mathrm{Cu}_{3} \mathrm{O}_{7-\delta}$ Thin Films on Yttria-Stabilized Zirconia Substrates with Crystalline Axes Tilted with Respect to the Surfaces, Physica C (Amsterdam) 275, 273 (1997).

[8] I. K. Bdikin, P. B. Mozhaev, G. A. Ovsyannikov, P. V. Komissinski, and I.M. Kotelyanskii, Growth and Domain Structure of $\mathrm{YBa}_{2} \mathrm{Cu}_{3} \mathrm{O}_{x}$ Thin Films and $\mathrm{YBa}_{2} \mathrm{Cu}_{3} \mathrm{O}_{x} / \mathrm{CeO}_{2}$ Heterostructures on Tilted $\mathrm{NdGaO}_{3}$ Substrates, Physica C (Amsterdam) 377, 26 (2002).

[9] P. B. Mozhaev, J.E. Mozhaeva, I. K. Bdikin, I. M. Kotelyanskii, V. A. Lusanov, J. Bindslev Hansen, C. S. Jacobsen, and A.L. Kholkin, Out-of-Substrate Plane Orientation Control of Thin $\mathrm{YBa}_{2} \mathrm{Cu}_{3} \mathrm{O}_{x}$ Films on $\mathrm{NdGaO}_{3}$ Tilted-Axes Substrates, Physica C (Amsterdam) 434, 105 (2006).

[10] M. Djupmyr, G. Cristiani, H.-U. Habermeier, and J. Albrecht, Anisotropic Temperature-Dependent Current Densities in Vicinal $\mathrm{YBa}_{2} \mathrm{Cu}_{3} \mathrm{O}_{7-\delta}$, Phys. Rev. B 72, 220507(R) (2005).

[11] C. A. Duran, P. L. Gammel, R. E. Miller, and D. J. Bishop, Observation of Magnetic-Field Penetration via Dendritic Growth in Superconducting Niobium Films, Phys. Rev. B 52, 75 (1995).

[12] M. Menghini, R.J. Wijngaarden, A. V. Silhanek, S. Raedts, and V. V. Moshchalkov, Dendritic Flux Penetration in $\mathrm{Pb}$ Films with a Periodic Array of Antidots, Phys. Rev. B 71, 104506 (2005).

[13] I. A. Rudnev, S. V. Antonenko, D. V. Shantsev, T. H. Johansen, A. E. Primenko, Dendritic Flux Avalanches in Superconducting $\mathrm{Nb}_{3} \mathrm{Sn}$ Films, Cryogenics 43, 663 (2003).

[14] I. A. Rudnev, D. V. Shantsev, T. H. Johansen, A.E. Primenko, Avalanche-Driven Fractal Flux Distributions in NbN Superconducting Films, Appl. Phys. Lett. 87, 042502 (2005).

[15] D. V. Denisov, D. V. Shantsev, Y.M. Galperin, Eun-Mi Choi, Hyun-Sook Lee, Sung-Ik Lee, A. V. Bobyl, P. E. Goa, A.A.F. Olsen, and T.H. Johansen, Onset of Dendritic Flux Avalanches in Superconducting Films, Phys. Rev. Lett. 97, 077002 (2006).

[16] S. C. Wimbush, B. Holzapfel, and Ch. Jooss, Magnetic Instability in $\mathrm{YNi}_{2} \mathrm{~B}_{2} \mathrm{C}$ Thin Films: Observation of Dendritic Flux Instabilities in Borocarbides, J. Appl. Phys. 96, 3589 (2004).

[17] E. Altshuler and T.H. Johansen, Colloquium: Experiments in Vortex Avalanches, Rev. Mod. Phys. 76, 471 (2004).

[18] A. L. Rakhmanov, D. V. Shantsev, Y. M. Galperin, and T.H. Johansen, Finger Patterns Produced by Thermomagnetic Instability in Superconductors, Phys. Rev. B 70, 224502 (2004).

[19] D. V. Denisov, A. L. Rakhmanov, D. V. Shantsev, Y. M. Galperin, and T.H. Johansen, Dendritic and Uniform Flux Jumps in Superconducting Films, Phys. Rev. B 73, 014512 (2006). 
[20] I. S. Aranson, A. Gurevich, M. S. Welling, R. J. Wijngaarden, V. K. Vlasko-Vlasov, V. M. Vinokur, and U. Welp, Dendritic Flux Avalanches and Nonlocal Electrodynamics in Thin Superconducting Films, Phys. Rev. Lett. 94, 037002 (2005).

[21] L.E. Helseth, R.W. Hansen, E. I. Il'yashenko, M. Baziljevich, and T.H. Johansen, Faraday Rotation Spectra of Bismuth-Substituted Ferrite Garnet Films with In-Plane Magnetization, Phys. Rev. B 64, 174406 (2001).

[22] P. E. Goa, H. Hauglin, Å. A. F. Olsen, M. Baziljevich, and T. H. Johansen, Magneto-Optical Imaging Setup for Single Vortex Observation, Rev. Sci. Instrum. 74, 141 (2003).

[23] For comparison, similar experiments were carried out on a sample with $\theta=0^{\circ}$. Here, the flux dynamics always developed quite smoothly, and difference images similar to that in panel 2(b) showed just featureless noise from the CCD camera.

[24] V. V. Yurchenko, D. V. Shantsev, T.H. Johansen, M. R. Nevala, I. J. Maasilta, K. Senapati, and R. C. Budhani, Reentrant Stability of Superconducting Films and the Vanishing of Dendritic Flux Instability, Phys. Rev. B 76, 092504 (2007).

[25] V. V. Yurchenko, T.H. Johansen, and Y.M. Galperin, Dendritic Flux Avalanches in Superconducting Films, Low Temp. Phys. 35, 619 (2009).

[26] Notice the very faint colors along the edge, which are caused by the flux jumps perturbing the external field near the edge and are a result of the nonlocal electrodynamics of thin-film superconductors [19,27].

[27] E.H. Brandt, and M. Indenbom, Type-II-Superconductor Strip with Current in a Perpendicular Magnetic Field, Phys. Rev. B 48, 12893 (1993); E. Zeldov, J. R. Clem, M. McElfresh, and M. Darwin, Magnetization and Transport Currents in Thin Superconducting Films, Phys. Rev. B 49, 9802 (1994).

[28] D. V. Shantsev, A. V. Bobyl, Y.M. Galperin, T. H. Johansen, and S.I. Lee, Size of Flux Jumps in Superconducting Films, Phys. Rev. B 72, 024541 (2005).

[29] R. Besseling, P. H. Kes, T. Dröse, and V. M. Vinokur, Depinning and Dynamics of Vortices Confined in Mesoscopic Flow Channels, New J. Phys. 7, 71 (2005).

[30] P. Bak, C. Tang, and K. Wiesenfeld, Self-Organized Criticality, Phys. Rev. A 38, 364 (1988).

[31] M. Bengrine, A. Benyoussef, F. Mhirech, and S. D. Zhang, Disorder-Induced Phase Transition in a One-Dimensional Model of Rice Pile, Physica A (Amsterdam), 272, 1 (1999).
[32] L. A. N. Amaral and K. B. Lauritsen, Energy Avalanches in a Rice-Pile Model, Physica A (Amsterdam) 231, 608 (1996).

[33] L.A. N. Amaral and K.B. Lauritsen, Self-Organized Criticality in a Rice-Pile Model, Phys. Rev. E 54, R4512 (1996).

[34] V. Frette, K. Christensen, A. Malthe-Sørensen, J. Feder, T. Jøssang, and P. Meakin, Avalanche Dynamics in a Pile of Rice, Nature (London) 379, 49 (1996).

[35] C.M. Aegerter, M.S. Welling, and R. J. Wijngaarden, Self-Organized Criticality in the Bean State of $\mathrm{YBa}_{2} \mathrm{Cu}_{3} \mathrm{O}_{7-x}$ Thin Films, Europhys. Lett. 65, 753 (2004).

[36] I. Guillamón, H. Suderow, S. Viera, J. Sesé, R. Córdoba, J. M. De Teresa, and M. R. Ibarra, Direct Observation of Stress Accumulation and Relaxation in Small Bundles of Superconducting Vortices in Tungsten Thin Films, Phys. Rev. Lett. 106, 077001 (2011).

[37] C. Prado and Z. Olami, Inertia and Break of SelfOrganized Criticality in Sandpile Cellular-Automata Models, Phys. Rev. A 45, 665 (1992).

[38] C. Reichhardt, C. J. Olson, J. Groth, S. Field, and F. Nori, Microscopic Derivation of Magnetic-Flux-Density Profiles, Magnetization Hysteresis Loops, and Critical Currents in Strongly Pinned Superconductors, Phys. Rev. B 52, 10441 (1995); Vortex Plastic Flow, Local Flux Density, Magnetization Hysteresis Loops, and Critical Current, Deep in the Bose-Glass and MottInsulator Regimes, Phys. Rev. B 53, R8898 (1996).

[39] K.E. Bassler and M. Paczuski, Simple Model of Superconducting Vortex Avalanches, Phys. Rev. Lett. 81, 3761 (1998).

[40] C. Reichhardt, J. Groth, C. J. Olson, S. Field, and F. Nori, Spatiotemporal Dynamics and Plastic Flow of Vortices in Superconductors with Periodic Arrays of Pinning Sites, Phys. Rev. B 54, 16108 (1996); C. J. Olson, C. Reichhardt, and F. Nori, Superconducting Vortex Avalanches, Voltage Bursts, and Vortex Plastic Flow: Effect of the Microscopic Pinning Landscape on the Macroscopic Properties, Phys. Rev. B 56, 6175 (1997).

[41] C. Reichhardt, C. J. Olson, and F. Nori, Commensurate and Incommensurate Vortex States in Superconductors with Periodic Pinning Arrays, Phys. Rev. B 57, 7937 (1998); Nonequilibrium Dynamic Phases and Plastic Flow of Driven Vortex Lattices in Superconductors with Periodic Arrays of Pinning Sites, 58, 6534 (1998). 